\title{
Clinical Spectrum of Movement Disorders in Neurology Inpatients in a Tertiary Care Centre
}

\author{
Shabeer Ahmad Paul ${ }^{1}$ Gouranga Prasad Mondal ${ }^{1}$ Ramesh Bhattacharyya ${ }^{1}$ \\ Kartik Chandra Ghosh ${ }^{1}$ Sarbajit Das ${ }^{1}$ Suman Das ${ }^{1}$ Hema Krishna ${ }^{1}$ Chandrakanta Patra ${ }^{1}$ \\ ${ }^{1}$ Department of Neurology, Calcutta National Medical College \& \\ Hospital, Kolkata, West Bengal, India

\begin{abstract}
Address for correspondence Shabeer Ahmad Paul, DM, Department of Neurology, Calcutta National Medical College \& Hospital, Kolkata 700014, West Bengal, India (e-mail: paulshabir55@gmail.com).
\end{abstract}

J Neurosci Rural Pract 2021;12:581-585.

\begin{abstract}
Background Little data are available on the spectrum of movement disorders in inpatients, particularly those admitted in neurology specialty. This may be related to the fact that patients presenting with movement disorders are usually evaluated from outpatient clinics.

Objective The aim of this study is to provide data on the pattern of movement disorders in neurology inpatients.

Materials and Methods Patients admitted through emergency department or neurology clinic with complaints of movement abnormalities were recruited in this study from October 2019 to September 2020. Cases were subjected to proforma-based detailed history, examination, and appropriate investigations.

Statistical Analysis Descriptive statistics using SPSS 20.

Results and Conclusion Bradykinesia with or without tremor was the most common movement disorder (28.3\%), followed by ataxia and dystonia (24.5\% each) and hemifacial spasm and myoclonus (7.5\% each). Chorea, tic disorder, and hemiballismus were also reported. Etiologies included Parkinson disease, Wilson's disease, subacute sclerosing panencephalitis (SSPE), drugs, stroke, spinocerebellar ataxia, Huntington's disease, neuroacanthocytosis, and others. Dystonia represented the most common

Keywords

- movement disorders

- inpatients

- spectrum disorder in the younger age group (44.4\%), whereas bradykinesia and/or tremor represented the most common movement disorder in the older age group (46.4\%). This study demonstrates the characteristic distribution of movement disorders in neurology inpatients.
\end{abstract}

\section{Introduction}

Movement disorders are neurological conditions characterized by an excess of movement or paucity of voluntary movement unrelated to weakness or spasticity. Movement disorders thereby fall into two broad categories-hyperkinetic and hypokinetic. Majority of the movement disorders present to outpatient clinics and can be evaluated and managed on outpatient basis only. However, there are circumstances when movement disorder cases are admitted and managed as inpatients. published online

May 10, 2021
DOI https://doi.org/

$10.1055 / \mathrm{s}-0041-1730086$ ISSN 0976-3147 (c) 2021. Association for Helping Neurosurgical Sick People.

This is an open access article published by Thieme under the terms of the Creative Commons Attribution-NonDerivative-NonCommercial-License, permitting copying and reproduction so long as the original work is given appropriate credit. Contents may not be used for commercial purposes, or adapted, remixed, transformed or built upon. (https://creativecommons.org/licenses/by-nc-nd/4.0/).

Thieme Medical and Scientific Publishers Pvt. Ltd. A-12, 2nd Floor, Sector 2, Noida-201301 UP, India 


\section{Materials and Methods}

In this study, patients presenting to emergency with acute onset movement disorders or chronic disease with acute exacerbation of symptoms were admitted to the neurology department. In addition, cases were also admitted through neurology clinic with a purpose of subjecting them to an extended evaluation, in order to reach an etiological diagnosis. This study was performed at the Calcutta National Medical College and Hospital from December 2019 to August 2020. A total of 53 cases were included. A detailed history and examination was done in all cases and was followed by the appropriate investigations to arrive at a specific diagnosis. The diagnosis of specific movement disorders like Parkinson's disease (PD), progressive supranuclear palsy (PSP), corticobasal degeneration (CBD), and normal pressure hydrocephalus (NPH) was achieved with the help of established criteria for these disorders. Data was analyzed using Microsoft Excel and SPSS version 20.

\section{Results}

The mean age of our patients was 44.31 years $(S D=19.76)$, ranging from 9 years to 75 years. Age characteristics of the study population is given in - Table 1 . Diabetes and hypertension were the two comorbidities noted specifically in elderly patients. The overall prevalence of movement disorders was higher in males (69.8\% vs. 30.2\% in females). Majority of our cases had a chronic onset (69.8\%). As much as $32.7 \%$ had subacute onset and $17 \%$ presented acutely.

Bradykinesia alone and in association with tremor was the most common movement disorder in our study, accounting for $28.3 \%$ of cases. This was followed by ataxia and dystonia (24.5\% each). The frequency of different presenting symptoms is shown in - Fig. 1.
Common etiological diagnosis included Parkinsonism, followed by stroke, SCA, and Wilson's disease. - Table 2 shows the frequency and percentage of different etiological conditions.

PD was the most common diagnosis among the patients presenting with bradykinesia with or without tremor, accounting for $73.3 \%$ of cases. PSP, CBD, NPH, and young Parkinsonism were the other diagnoses.

Dystonia was mostly focal and segmental, with limb dystonia being the most common distribution, followed by cervical. Wilson's disease was the most common etiology seen in 4/13 cases. Frequency of various causes of dystonia is shown in - Fig. 2. Etiology was undefined in 5/13, which mostly represented the older age group. Limb dystonia was the most common pattern of dystonia, followed by cervical, as depicted in - Fig. 3. Among the Wilson's disease patients, Kayser-Fleischer (KF) ring was seen in $75 \%$ ( $3 / 4$ cases), and liver involvement and mental retardation were seen in $50 \%$ each (2/4 cases). Overall, dystonia was more common in males (11/13 cases). Dystonia was acute onset in 2/13 cases, subacute in $2 / 13$, and chronic in 9/13 cases.

Ataxia was acute in 46.2\% (6/13 cases), subacute in 1/13, and chronic in $6 / 13$ cases. Stroke represented the most common etiology, with infarct seen in 4/13 cases and hemorrhage in $1 / 13$ cases. SCA was diagnosed to be the etiology in $4 / 13$ cases, with two cases documented to have SCA-2 on genetic studies. - Fig. 4 shows the distribution of different causes of ataxia.

Tremor in our cases was not seen in isolation. It tended to occur in combination with other movement disorders, most commonly with bradykinesia and some cases of ataxia, being resting and postural type in the former and intentional in the latter. Hemifacial spasm was seen in four cases, all being female. MRI in all the cases was normal. All four cases showed a good response to botulinum toxin injection.

Table 1 Age statistics in different movement disorders

\begin{tabular}{|l|l|l|l|l|l|}
\hline Movement disorder & Number & Minimum & Maximum & Mean & Standard deviation \\
\hline Dystonia & 13 & 9 & 60 & 31.92 & 16.348 \\
\hline Bradykinesia/Tremor & 15 & 25 & 75 & 61.07 & 12.475 \\
\hline Ataxia & 13 & 17 & 62 & 48.62 & 14.448 \\
\hline Myoclonus & 4 & 14 & 55 & 25.75 & 19.568 \\
\hline Hemifacial spasm & 4 & 24 & 54 & 40.75 & 12.579 \\
\hline
\end{tabular}

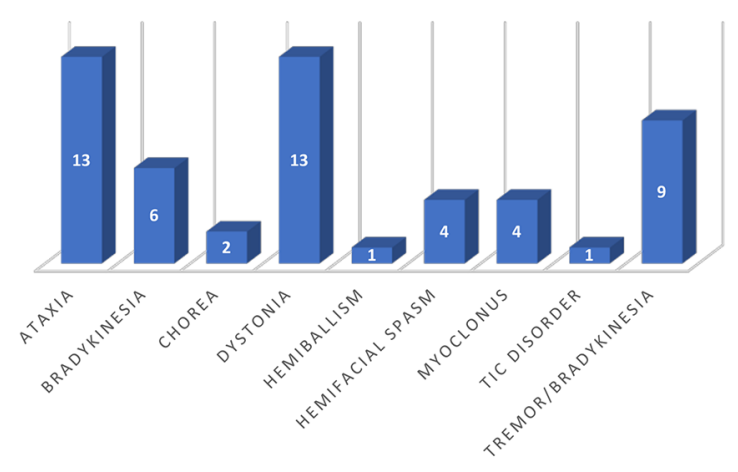

Fig. 1 Frequency of movement disorder symptoms.

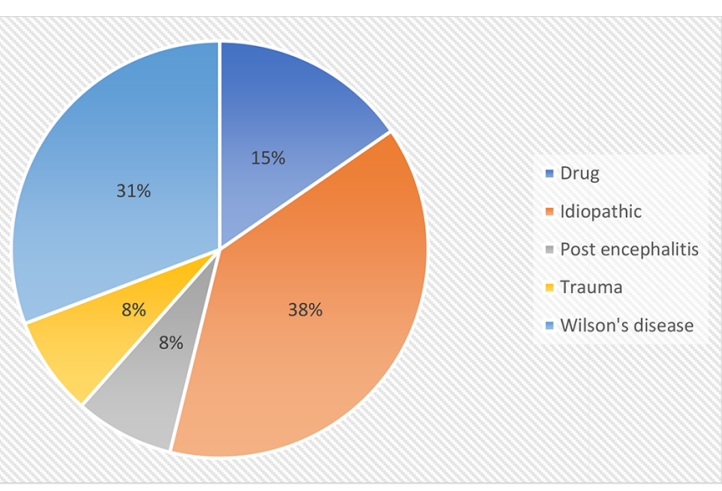

Fig. 2 Frequency of different etiologies of dystonia. 
Table 2 Frequency of different etiological diagnosis

\begin{tabular}{|l|l|l|}
\hline Etiology & Frequency & Percentage \\
\hline Alcoholism & 1 & 1.9 \\
\hline CBD & 1 & 1.9 \\
\hline ICH & 1 & 1.9 \\
\hline CJD & 1 & 1.9 \\
\hline Drug & 2 & 3.8 \\
\hline Friedreich's ataxia & 1 & 1.9 \\
\hline Huntington's disease & 1 & 1.9 \\
\hline Idiopathic & 6 & 11.32 \\
\hline Hemifacial spasm & 4 & 7.54 \\
\hline Infarct & 5 & 9.4 \\
\hline MS & 1 & 1.9 \\
\hline MSA-C & 1 & 1.9 \\
\hline Neuroacanthocytosis & 1 & 1.9 \\
\hline NPH & 1 & 1.9 \\
\hline PD & 11 & 20.8 \\
\hline Postencephalitis & 1 & 1.9 \\
\hline PSP & 1 & 1.9 \\
\hline SCA & 4 & 7.5 \\
\hline SSPE & 3 & 5.7 \\
\hline Trauma & 1 & 1.9 \\
\hline Wilson's disease & 4 & 7.5 \\
\hline Young Parkinsonism & 1 & 1.9 \\
\hline Total & 53 & 100 \\
\hline Abrerions: & 1 & \\
\hline
\end{tabular}

Abbreviations: CBD, corticobasal degeneration; CJD, Creutzfeldt-Jakob disease; ICH, intracerebral hemorrhage; MS, multiple sclerosis; MSA$\mathrm{C}$, multiple system atrophy-cerebellar subtype; NPH, normal pressure hydrocephalus; PD, Parkinsonian disease; PSP, progressive supranuclear palsy; SCA, spinocerebellar ataxia; SSPE, subacute sclerosing panencephalitis.

Myoclonus was seen in four cases. SSPE was diagnosed with the help of immunoglobulin G (IgG) antimeasles antibody and cerebrospinal fluid (CSF)/serum IgG antimeasles antibody ratio, which was demonstrated to be high in all three cases. EEG showed classical $6 \mathrm{~Hz}$ periodic complexes in all three cases. All presented within 3 to 6 months of onset of symptoms. One patient was treated with intraventricular interferon using ommaya reservoir. However, the patient expired after showing an initial response. Creutzfeldt-Jakob disease (CJD) was diagnosed in the elderly patient, with the help of clinical, radiological, and EEG criteria.

Movement disorders had a different pattern in the younger and older age groups, with dystonia being more common in the former and bradykinesia with or without tremor in the latter as depicted in - Fig. 5 .

Among the acute onset movement disorders, ataxia was the most common followed by dystonia, whereas among the chronic onset disorders, bradykinesia alone or in combination with tremor was the most common followed by ataxia and dystonia. Subacute onset represented a mixture of different etiologies as demonstrated in - Fig. 6.

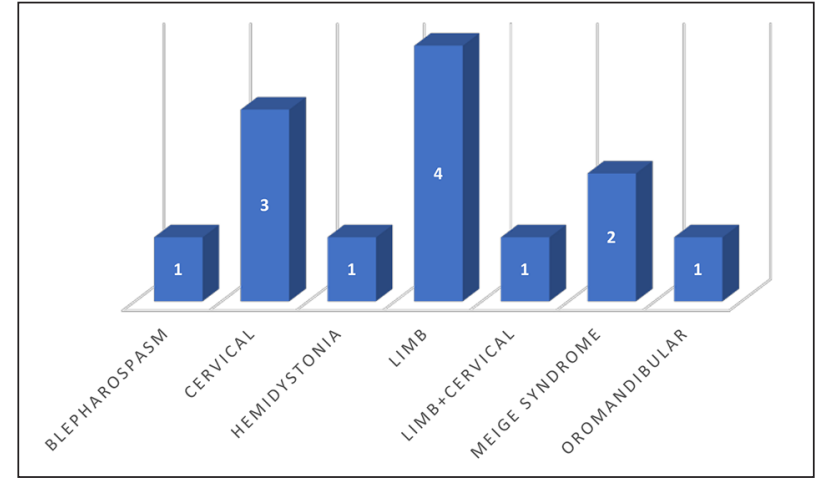

Fig. 3 Distribution of different patterns of dystonia.

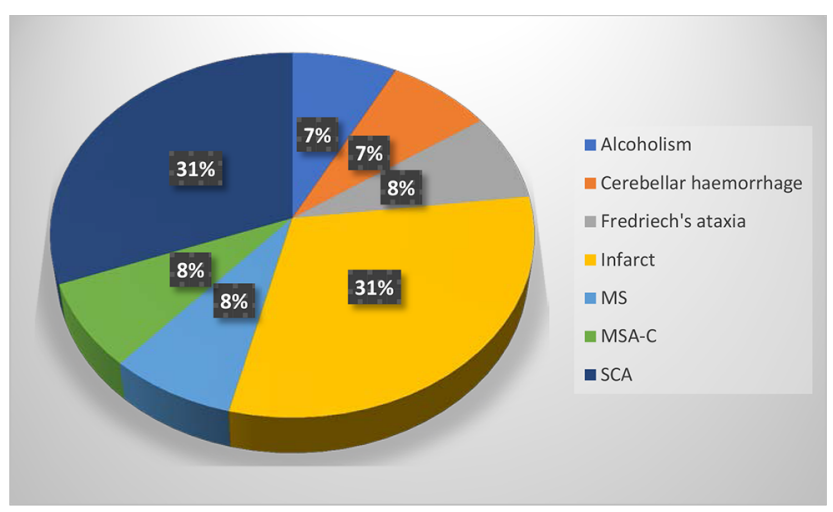

Fig. 4 Frequency of different causes of ataxia.

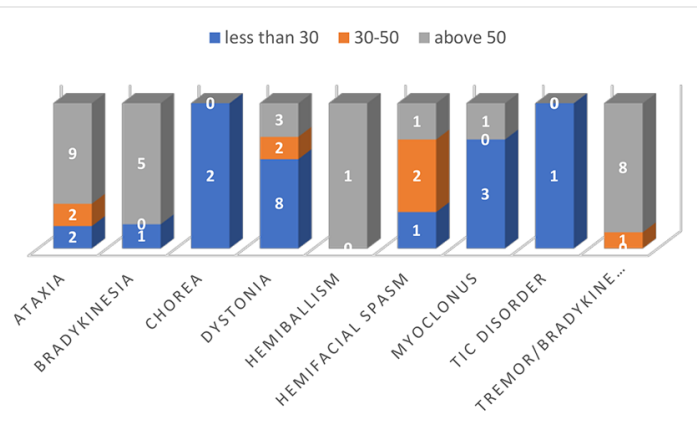

Fig. 5 Distribution of different movement disorder symptoms with respect to age group.

\section{Discussion}

The study was intended to find the spectrum of movement disorders admitted at a tertiary care hospital in the neurology department. Previous studies have mainly focused on cases attending the outpatient clinics, ${ }^{1,2}$ although there have been studies on spectrum of movement disorders in pediatric inpatients. ${ }^{3}$ A study was also conducted by Kwon et al on clinical characteristics of movement disorders in inpatients as part of neurologic consultations from other departments. ${ }^{4}$ However, not much literature is available on the spectrum of movement disorders in inpatients admitted specifically in the neurology department. The purpose of 


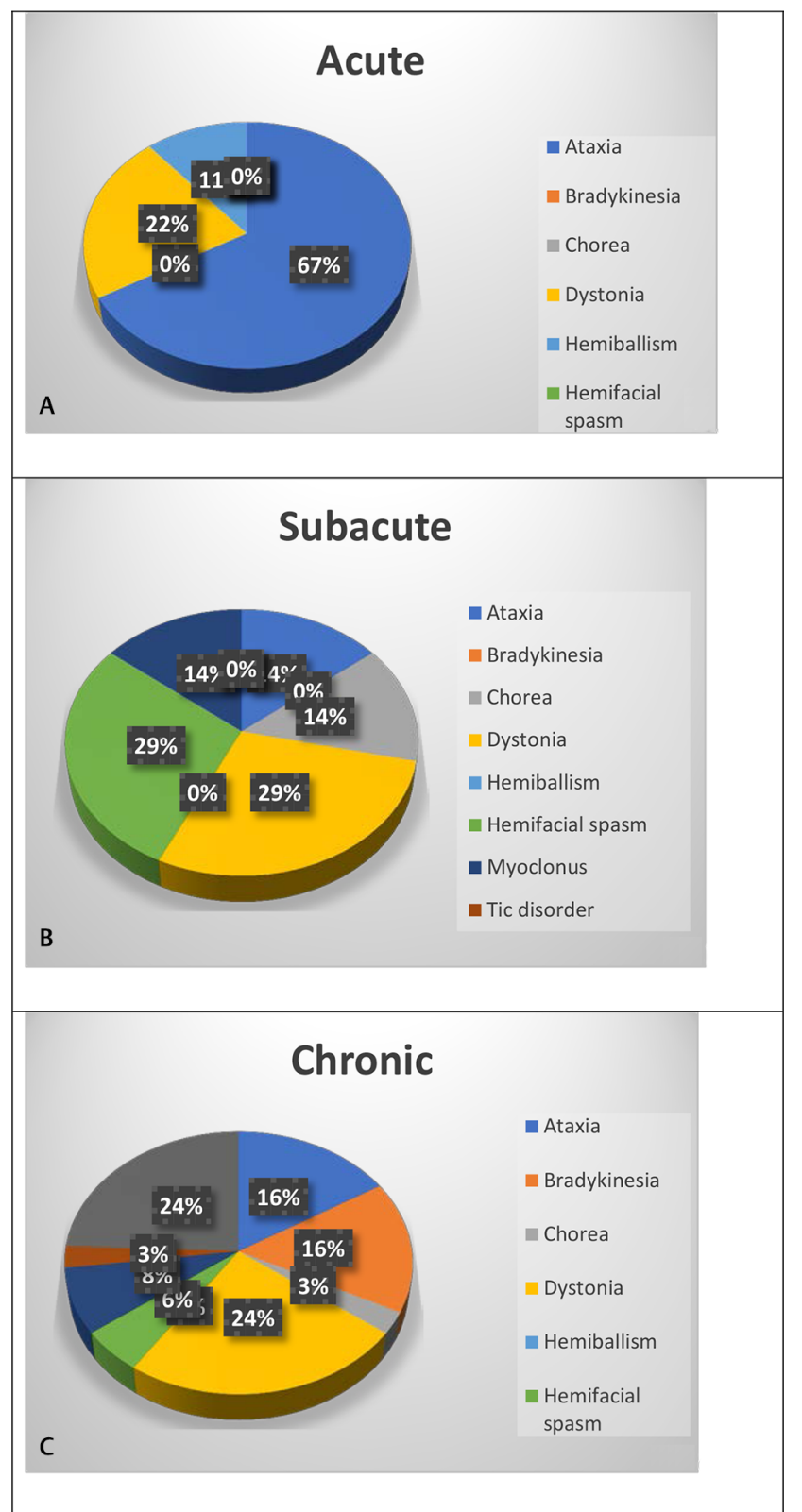

Fig. 6 Distribution of various movement disorders with regard to onset $(\mathbf{A})$ acute onset $(\mathbf{B})$ subacute onset $(\mathbf{C})$ chronic onset.

admission may include detailed evaluation of movement disorder or inpatient therapeutic management in case of acute conditions or as part of the teaching program for neurology residents. Therefore, spectrum of movements disorders may present a different pattern compared with outpatient studies.

Our study reported bradykinesia with or without tremor to be the most common movement disorder, followed by dystonia and ataxia. In contrast, tremor was reported to be the most common symptom, followed by myoclonus, in non-neurological patients in the study by Kwon et al. In our study, however, which focused only on neurological patients, tremor occurred as an associated symptom; resting tremor in association with bradykinesia and intentional tremor in association with ataxia. Essential tremor was not reported in our study, as it is usually managed on an outpatient basis.
We found that dystonia was idiopathic in $38 \%$ of cases. This may be because most of the dystonia cases in middle aged and elderly do not undergo genetic testing because of nonavailability of such facilities in majority of the health care centers and high cost of dystonia genetic testing panel. In a study on focal dystonia in children in a tertiary care center, Rupesh et al reported $78.6 \%$ cases to be idiopathic. ${ }^{3}$ Among the secondary causes of dystonia, the same author reported drugs to be the most common cause followed by birth insult. In a case study of 83 patients of cervical dystonia by Camargo et al, $62.3 \%$ were reported to be idiopathic, followed by tardive dystonia in $8.2 \%$ and familial in $5.9 \% .^{5}$ Pandey et al reported dystonia in $24.7 \%$ of cases in his study on movement disorders in children on outpatient basis. ${ }^{1}$ Andrade et al reported $46.2 \%$ to have secondary dystonia, with tardive dystonia being the most common etiology followed by perinatal cerebral injury. ${ }^{6}$

In our study, Wilson's disease constituted $30.8 \%$ of dystonia cases, and drugs were the etiology in $15 \%$. Limb and cervical dystonia were equally common (30.7\% each), followed by blepharospasm with or without oromandibular dystonia (23.07\%). Studies have reported limb dystonia to be more common in younger age group and cranial dystonia in older age group. ${ }^{3}$ The limited number of cases in our study makes it difficult to lend support to any such assumption.

Our study revealed that stroke represented the most common etiology in acute onset movement disorders, whereas PD was the most common etiology for disorders with chronic onset. The mean age of presentation in PD patients was 64.09 years (standard deviation [SD] = 7.21 years), which is comparable, with 60 years being the mean age of presentation reported in Western literature. ${ }^{7}$ The time to diagnosis ranged from 180 days to 610 days before presentation, with a mean of 403 days. This represents somewhat delayed seeking of medical advice for PD symptoms in patients from developing countries compared with data from Western literature, which shows mean duration from symptom onset to diagnosis being 9 months. ${ }^{8}$

Among the ataxia patients, degenerative etiology superseded other causes and was seen in $46.15 \%$, while stroke was the most common etiology among the secondary causes. Four cases were diagnosed to have SCA, two among them detected to be SCA-2, and one SCA-3 on genetic testing. Apart from SCA, one case was diagnosed to be Friedreich's ataxia and one multiple system atrophy-cerebellar subtype (MSA-C). In a large study of 1500 ataxia patients, Hadjivassiliou et al found that gluten-sensitive ataxia was the most common cause of sporadic ataxia, followed by genetic and MSA-C, whereas in the familial group, Friedreich's ataxia was reported in $22 \%$, followed by SCA- 6 in $14 \%$ and episodic ataxia-2 (EA-2) in 13\%. SCA-2 happens to be the most common SCA variant in India and SCA-3 worldwide. ${ }^{9}$

In our study, we found SSPE accounting for $75 \%$ of myoclonus cases. Although the prevalence of SSPE has reduced to 1 to 2 per million cases of measles in developed nations, but it continues to be significantly high in developing countries. ${ }^{10}$ One study from India in 2008 reported 458 cases of 
SSPE over a 10-year period. ${ }^{11}$ Cases of SSPE have been reported even in individuals who are vaccinated for measles. ${ }^{12}$

Hemifacial spasm (HFS) in our study occurred exclusively in females with mean age of 41 years. Females are known to be more prone to develop HFS with a ratio of $2: 1 .{ }^{13}$ Wing Lok et al reported two-thirds of the cases to be females in his case series of 137 HFS patients. ${ }^{14}$ All four patients in our study needed botulinum toxin in addition to other medications and showed a good response.

Chorea was reported with a low frequency of $2 / 53$ (3.77\%) in our study. Etiology was established as Huntington's disease and neuroacanthocytosis. This is comparable to the prevalence of chorea reported in other studies, as reported by Baumer et al, in childhood onset movement disorders. ${ }^{15}$ Sydenham's chorea is reported to be the most common cause of chorea in children and Huntington's disease in adults..$^{16}$ Our study did not report any case of Sydenham's chorea as majority of our cases belonged to the adult age group. Strikingly, we did not encounter any case of movement disorder with autoimmune etiology, alluding to the rare nature of this distinct etiologic subgroup.

Our study was limited by a small number of cases and included a spectrum of patients with different indications of admission not necessarily limited to the acute presentation.

\section{Conclusion}

Our study showed a characteristic pattern of movement disorders in a specific group of inpatients admitted under neurology care. Bradykinesia with or without tremor constituted the most common movement disorder, followed by ataxia and dystonia. Etiologies included PD and Parkinson plus syndromes, Wilson's disease, SSPE, drugs, stroke, SCA, Huntington's disease, neuroacanthocytosis, and others.

\section{Ethical Approval}

All procedures performed in studies involving human participants were in accordance with the ethical standards of the institutional and/or national research committee and with the 1964 Helsinki Declaration and its later amendments or comparable ethical standards. Informed consent was obtained from all individual participants involved in the study.

\section{Authors' Contributions}

G.P. Mondal and S.A. Paul developed the concept. R. Bhattacharyya designed the study. Sarbajit Das, Suman Das, H. Krishna, and C. Patra collected the data. K.C. Ghosh conducted literature overview and was involved in discussion. S.A. Paul analyzed the data and performed the statistical analysis.
Funding

None.

\section{Conflict of Interest}

None declared.

\section{References}

1 Pandey CS, Kumar R, Malhotra HS, Kohli N, Kumar C, Verma SK. Movement disorders in children: an observational study in a tertiary care center in north India. J Pediatr Neurol 2020;18(3):135-140

2 Eu KM, Tan LCS, Tan ARJ, et al. Spectrum and burden of movement disorder conditions in a tertiary movement disorders centre-a 10-year trend. Ann Acad Med Singap 2014;43(4):203-208

3 Prasad R, Joshi DD, Mishra V, Chaurasia R, Pathak A. Clinical spectrum of focal dystonias: experience from a Tertiary Care Center. Ann Mov Disord 2020;3(2):99

4 Kwon K-Y, Lee HM, Lee S-M, Koh S-B. Clinical characteristics of involuntary movement in hospitalized patients. J Mov Disord 2019;12(1):31-36

5 Camargo CHF, Teive HAG, Becker N, Baran MHH, Scola RH, Werneck LC. Cervical dystonia: clinical and therapeutic features in 85 patients. Arq Neuropsiquiatr 2008;66(1):15-21

6 Andrade LA, Ferraz HB. Idiopathic dystonia. Clinical profile of 76 Brazilian patients. Arq Neuropsiquiatr 1992;50(4):426-432

7 de Lau LML, Breteler MM. Epidemiology of Parkinson's disease. Lancet Neurol 2006;5(6):525-535

8 Saunders-Pullman R, Wang C, Stanley K, Bressman SB. Diagnosis and referral delay in women with Parkinson's disease. Gend Med 2011;8(3):209-217

9 Sinha KK, Worth PF, Jha DK, et al. Autosomal dominant cerebellar ataxia: SCA2 is the most frequent mutation in eastern India. J Neurol Neurosurg Psychiatry 2004;75(3):448-452

10 Jafri SK, Kumar R, Ibrahim SH. Subacute sclerosing panencephalitis - current perspectives. Pediatric Health Med Ther 2018;9:67-71

11 Sonia M, Lalit D, Shobha B, et al. Subacute sclerosing panencephalitis in a tertiary care centre in post measles vaccination era. J Commun Dis 2009;41(3):161-167

12 Jagtap SA, Nair MD, Kambale HJ. Subacute sclerosing panencephalitis: A clinical appraisal. Ann Indian Acad Neurol 2013;16(4):631-633

13 Chopade TR, Bollu PC. Hemifacial Spasm. Available at: http://www.ncbi.nlm.nih.gov/books/NBK526108/. Accessed November 1, 2020

14 Au W-L, Tan LC, Tan AK. Hemifacial spasm in Singapore: clinical characteristics and patients ' perceptions. Ann Acad Med Singap 2004;33(3):324-328

15 Bäumer T, Sajin V, Münchau A. Childhood-onset movement disorders: a clinical series of 606 cases. Mov Disord Clin Pract (Hoboken) 2016;4(3):437-440

16 Bhidayasiri R, Truong DD. Chorea and related disorders. Postgrad Med J 2004;80(947):527-534 